\title{
PON Topologies for Dynamic Optical Access Networks
}

\author{
Pandelis Kourtessis, Member, IEEE, Yuval Shachaf, Ching -H. Chang, John M. Senior \\ Optical Networks Group, Science and Technology Research Institute (STRI), University of Hertfordshire \\ College Lane Campus, Hatfield, AL109AB, UK.+44 (0) 1707285082.p.kourtessis@herts.ac.uk
}

\begin{abstract}
PON-based access networks envisage the demonstration of scalability to allow gradual deployment of time and wavelength multiplexed architectures in a single-platform without changes in fibre infrastructure and also highly-efficient bandwidth allocation for service provision and upgrade on-demand. This is achieved by the application of coarse-fine grooming to route reflective-ONUs of time and wavelength PONs collectively and the development of MAC protocols enabling the OLT to dynamically assign available time slots among ONUs and demonstrate efficient bandwidth and wavelength assignment.
\end{abstract}

Keywords: PON, coarse WDM (CWDM), dynamic bandwidth allocation (DBA), quality of service (QoS).

\section{INTRODUCTION}

The emergence of new bandwidth-intensive applications articulated by distance learning, online gaming, Web 2.0 and movie delivery by means of high-definition video, has ultimately justified the necessity of upgrading the access network infrastructure to provide fat-bandwidth pipelines at subscriber close proximity. Passive optical networks (PONs) offer currently more opportunities to communicate these services than ever before, with potential connection speeds of up to $100 \mathrm{Mbit} / \mathrm{s}$ in mind [1]. A scalable multi-PON access network architecture [2] has been investigated in that direction to provide interoperability among dynamic time division multiplexing (TDM) and wavelength division multiplexing (WDM)-PONs through coarse WDM (CWDM) routing in the optical line terminal (OLT). To provide bandwidth on demand, a novel TDM dynamic minimum bandwidth (DMB) allocation protocol and an upgraded version have been proposed to achieve quality of service (QoS) at three different service levels and diverse network throughputs [3]. In addition, to allow for WDM-PON resource allocation, to overcome the inevitable network congestion of single wavelength networks, the developed medium access control (MAC) protocols have been extended to implement logical point-to-point topologies based on general loop-back WDM-PON architectures [2] to increase service provisioning between reflective optical network units (ONUs) and the OLT by vigorously distributing network capacity simultaneously between the upstream and downstream.

\section{NETWORK ARCHITECTURE}

The network architecture in Fig. 1 exhibits a single $4 \times 4$ coarse array waveguide grating (AWG) in the OLT to route multiple TDM and WDM-PONs by means of a single tunable laser (TL1) and receiver (RX1) allowing for coarse-fine grooming to display smooth network upgrade. Proposed coarse AWG devices display $7 \mathrm{~nm}, 3 \mathrm{~dB}$ Gaussian passband windows [4], denoted in Fig. 1 by coarse ITU-T channels $\lambda_{1}=1530 \mathrm{~nm}$ and $\lambda_{2}=1550 \mathrm{~nm}$, set to accommodate up to $16,0.4 \mathrm{~nm}$-spaced wavelengths to address a total of 16 ONUs per PON. In downstream, TL1 will optimally utilise $\lambda_{1}{ }^{9}$, placed at the centre of the AWG coarse channel $\lambda_{1}$, to broadcast information to all ONUs of TDM-PON1. To address a WDM-PON, TL1 will switch on all 16 wavelengths, centred $\pm 3.2 \mathrm{~nm}$ around coarse channel $\lambda_{2}$, i.e. $\lambda_{2}^{1-16}$, to address jointly all ONUs in WDM-PON4 [5].

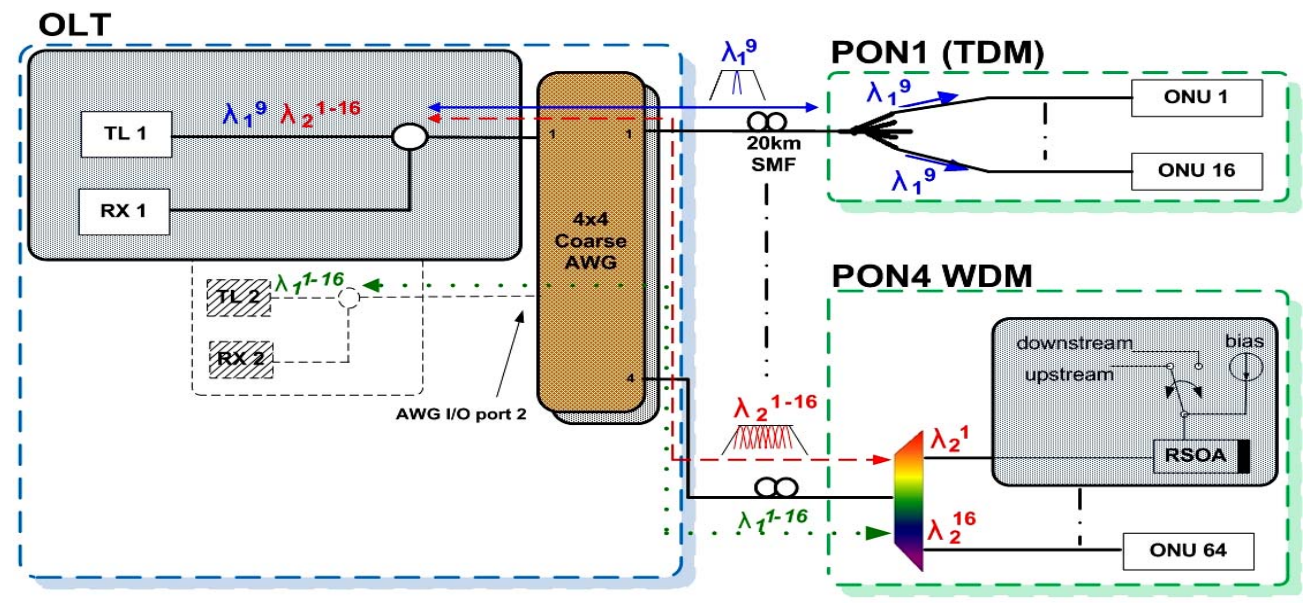

Figure 1. Unlimited-capacity multi-PON access network architecture. 
The established network interoperability is a key feature since it allows a smooth migration from single to multiwavelength optical access to address increasing bandwidth requirements. Reflective semiconductor optical amplifier (RSOA)-based ONUs are universally employed, avoiding the necessity of wavelength-specific, local optical sources. The use of multiple transceivers in a single OLT to serve all reflective PONs [5] allows for centralised control to distribute ONU capacity among upstream and downstream on demand and concurrently provide each PON with multiple wavelengths for enhanced bandwidth allocation flexibility. Finally, the network exhibits increased scalability since extra TLs can be directly applied at unused AWG ports in the OLT, e.g. TL2 at I/O port 2, to maintain high network performance at increased traffic load with low OLT inventory count since the ratio of subscriber number to OLT transceivers is comparatively high.

\subsection{Network routing performance}

In defining accurate routing of all ONUs comprising a PON, the AWG polarisation-dependent wavelength (PDW) shift [4] and associated polarisation-dependent loss (PDL) have been simulated and also experimentally demonstrated. It has been previously established that PDW shift of up to $1.8 \mathrm{~nm}$ could potentially impose a restriction in multi-wavelength operation over wide-passband AWG channels and therefore limit the routing performance of the entire network [2,5]. Figure 2(a) displays the measured PDL versus operating wavelength at PDW shifts of $0,1.5$ and $1.8 \mathrm{~nm}$ using VPI physical layer simulation platform. The vertical lines represent the $\pm 3.2 \mathrm{~nm}$ spectral width required for transmission of all wavelengths in a PON. As becomes evident from the figure, the PDL varies from $0 \mathrm{~dB}$ at centre wavelength to more than $5 \mathrm{~dB}$ outside the transmission band. As expected, the $0 \mathrm{~nm}$ shift curve exhibits insignificant residual PDL due to the random phase errors in the AWG channel. On the contrary, the PDL for the $1.5 \mathrm{~nm}$ and $1.8 \mathrm{~nm}$ curves demonstrate asymmetric, linearly increasing loss with regard to the centre wavelength since, due to shifting, the longer wavelengths suffer extended PDL. It can be further observed that the longest wavelength in the passband, at $\lambda_{2}{ }^{1}=1553.33 \mathrm{~nm}$, experiences the highest PDL with approximately $2.5 \mathrm{~dB}$ more loss than the central wavelength at $\lambda_{2}{ }^{9}=1550.12 \mathrm{~nm}$.

To demonstrate the effect of passband shifting and loss superimposed at the transmitted wavelengths, Fig. 2(b) displays the PDW shift of the central channel $\lambda_{2}=1550 \mathrm{~nm}$, for which the transverse magnetic (TM) response is $-1.8 \mathrm{~nm}$ shifted [4] with respect to the transverse electric (TE). It can be observed that on top of the $5 \mathrm{~dB}$ insertion loss of the channel, the longest wavelength at $\lambda_{2}{ }^{1}=1553.33 \mathrm{~nm}$ can suffer up to $5.5 \mathrm{~dB}$ loss, compared to the central wavelength at $\lambda_{2}{ }^{9}=1550.12 \mathrm{~nm}$, due to the increase of PDL and the AWG passband Gaussian response. To evaluate the bidirectional network performance, bit-error-rate (BER) responses have been recorded [5] for the least and most severely degraded ONUs of WDM-PON4. Results confirmed error-free transmission with measured $10^{-9}$ BER achieved by $93 \%$ of ONUs when $1.8 \mathrm{~nm}$ PDW is employed. Further investigations into the highest possible PDW to allow all ONUs achieve a BER of $10^{-9}$ produced a PDW figure of $0.8 \mathrm{~nm}$.
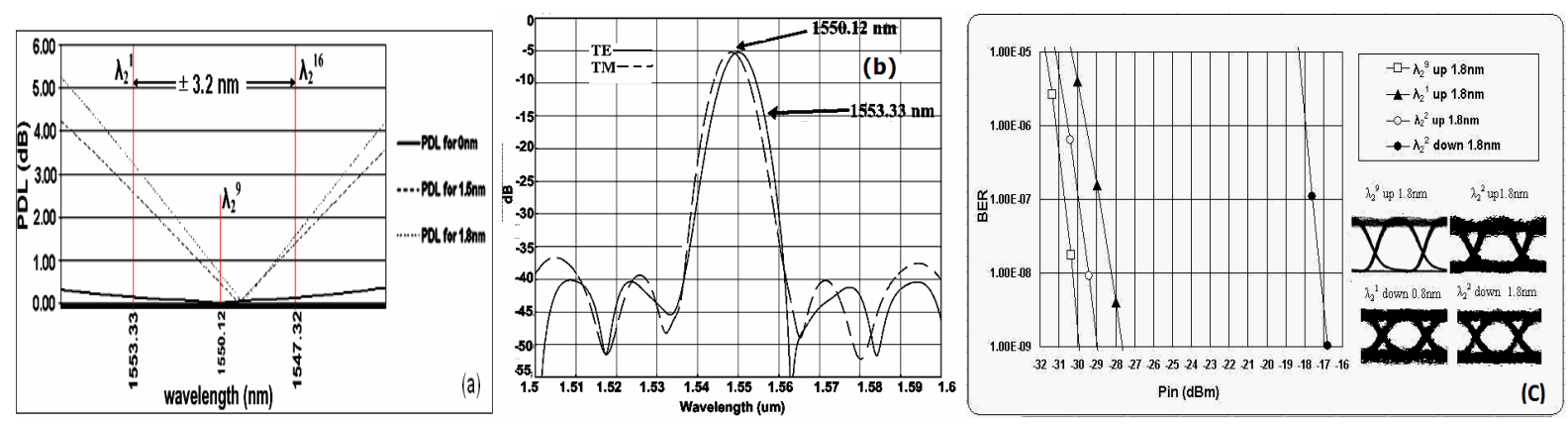

Figure 2. (a)PDL versus operating wavelength;(b)PDW of central channel;

(c) BER down/upstream transmissions.

\section{NETWORK BANDWIDTH MANAGEMENT}

To allow centralised, dynamic bandwidth allocation among the architecture's TDM and WDM-PONs, the OLT according to the developed algorithms will assign varying frame time-slots, initially to each PON in order-ofdemand and subsequently arrange each PON bandwidth among their ONUs based on their service level and individual bandwidth requirement $[3,6]$. In that direction, the DMB protocol [3] provides ONUs in TDM-PONs with three service levels at different weights, $W_{t}$, to represent network accessing priority. Subsequently the algorithm automatically assigns to each ONU a guaranteed minimum bandwidth, $B^{t}{ }_{\min }$, from the overall network capacity, to satisfy their basic service requirements at various service levels $t$ and apportions any unused bandwidth to ONUs according to their buffer queuing status. Following probable variations in network capacity, it is capable of readjusting the guaranteed minimum and unused bandwidths among ONUs to comply with subscriber contracts. For service level $t$, for example the maximum allocated bandwidth $B_{\text {max_allocated }}$ for $\mathrm{ONU}_{i}$ 
will be equal to the addition of $B_{\text {min }}^{t}$ and the extra assigned bandwidth, $B_{\text {ex_assigned. }}$ Otherwise, if the bandwidth requirement, $R_{i}$, is smaller than the total, $B_{\text {max_allocated }}$ will be equal to the required bandwidth, $R_{i}$, as given by (1).

$$
B_{\text {max_allocated }}^{i}=\min \left(\begin{array}{l}
B_{\min }^{t}+B_{e x_{-} \text {assigned }}^{i} \\
R_{i}
\end{array}\right)
$$

To further reduce the packet waiting time in the ONUs, the network traffic self-similarity characteristics have been incorporated into the DMB protocol [6]. In addition, since the upstream and downstream channels are independent, the grant massages for subsequent polling cycles can be communicated before the last ONU has finished its upstream transmission. Consequently the OLT in what is known as the advanced DMB (ADMB) protocol possess the capability to automatically re-arrange the upstream transmission order by assigning the ONU with the longest upstream transmission period to the last upstream time slot, reducing the idle period and increasing the overall network throughput. Contrasting the ADMB protocol with published dynamic bandwidth assignment algorithms, simulation results have shown [6] substantial reductions in mean packet delay, particularly at high network load in relation to the IPACT algorithm [7]. As presented in Fig. 3(a) it is also demonstrated that by adjusting the effective upstream transmission order of the network ONUs, the maximum network throughput in the ADMB protocol can be significantly increased to fully utilise the available bandwidth for different traffic loads.

Progressively, a WDM bandwidth allocation protocol has also been investigated to control simultaneously upstream and downstream data transfer. In accordance with the reflective multi-wavelength DMB (MDMB) protocol, OLT frames display time slots occupied by either downstream data or un-modulated continuous waves (CWs) and unlike TDM-PON algorithms, where only the ONU upstream time slots are considered, each ONU bandwidth requirement, $R_{i}$, as given in (1) should be replaced by their bidirectional bandwidth requirement, $R_{i}^{\prime}$, to account for both upstream and downstream. In addition the OLT assigned bandwidth, $B_{\text {allocated }}^{i}$, should be consequently distributed between the upstream and downstream according to individual demand.

The upstream channel throughput characteristics for the contrasted protocols are shown in Fig. 3(b). According to the WDM_528 M_down response, representing the MDMB protocol throughput distribution for a typical $528 \mathrm{Mbit} / \mathrm{s}$ downstream service demand, matching current TDM aggregate downstream rates, the recorded upstream throughput figure corresponds to approximately $1.6 \mathrm{Gbit} / \mathrm{s}$. This translates to $128 \% \mathrm{upstream}$ network efficiency compared to $94 \%$ achieved with the DMB protocol for TDM PONs.
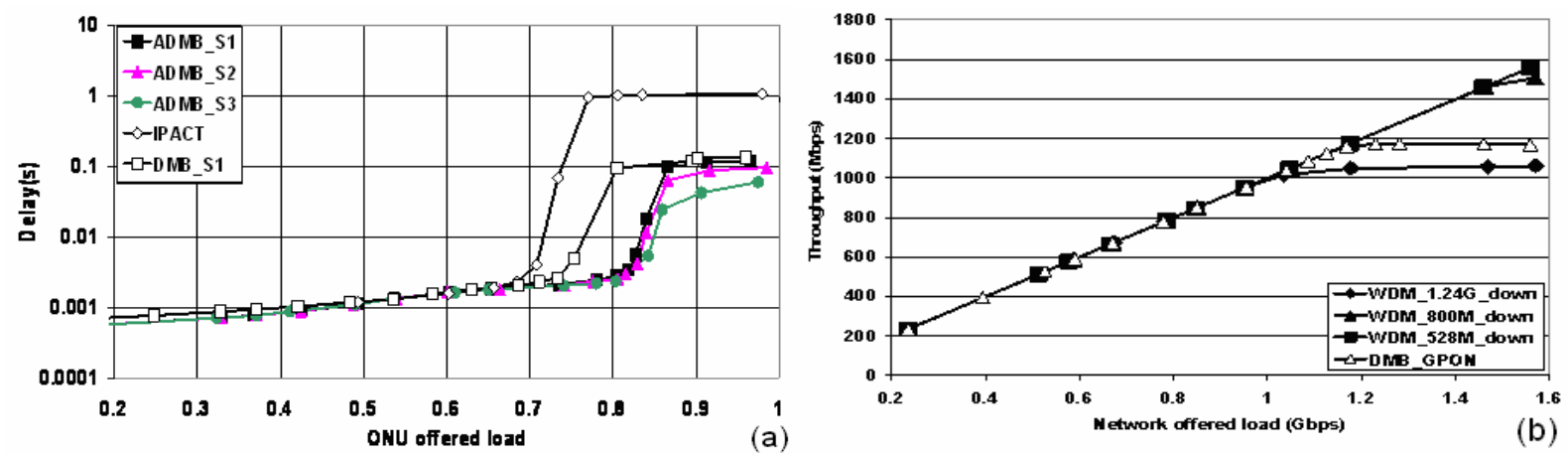

Figure 3. (a) Mean packet delay in IPACT, DMB and ADMB; (b) upstream throughput in DMB and MDMB.

In parallel the standard GPON upstream and downstream frame maps are currently explored to support multiwavelength operation, in the presence of four service levels. To conform to standard GPON architectures with the intention of reducing implementation costs, both the G.984.1 and G.694.2 ITU-T standards have been considered in a new protocol, consequently designed to initially accommodate a maximum five possible upstream wavelengths. To support varying bandwidth requirements at increased subscriber numbers, resource allocation utilises the already developed algorithms to sustain DBA with service level agreement at the time domain and also ONU wavelength sharing priority in the wavelength domain.

\section{LONG-REACH PONS}

The scalability of the presented network architecture is currently further explored by investigating its ability to demonstrate a long-reach, wide-splitting ratio network shared by numerous TDM and WDM-PONs [8], offering significant cost savings in an incorporated access-metro network infrastructure [8]. The current architecture is believed to be straightforwardly extendable to such a topology since it already serves a number of different physical PON locations with dedicated coarse wavelength channels. To that extend, extensive splitting ratios can be achieved by exploiting the decentralised coarse routing capability of the architecture to allow amplification units to be installed on route if necessary and to allocate increased number of dedicated wavelength channels to each physical PON using the multiple free spectral ranges (FSRs) of the CWDM AWG. 
In view of the MAC layer of a typical $100 \mathrm{~km}$ long-reach TDM-PON, the direct implementation of the DMB protocol has displayed limited performance in terms of bandwidth utilisation due to a recorded, $500 \mu$ s increase in packet propagation time compared to $25 \mathrm{~km}$-PONs, exhibiting a total of $1000 \mu \mathrm{s}$-wide idle time-slots in each transmission cycle as a result of the report and grant packets polling times. To achieve acceptable channel throughput and packet delay performance, an innovative two-state DMB (TSD) protocol has been demonstrated that utilises the idle time slots in the sense of virtual polling cycles, during which the ONUs can transmit data by means of a prediction method estimating their bandwidth requirement [9]. The amount of virtual bandwidths, allocated to ONUs, are determined by the DMB algorithm with each estimated ONU bandwidth requirement and $1000 \mu \mathrm{s}$ idle period regarded as the maximum polling period parameter [9]. As a result, simulation results have presented a significant $34 \%$ improvement in terms of channel throughput performance as well as a reduction in packet delay and packet loss rate [9].

\section{HYBRID WIRELESS OPTICAL NETWORKS}

The need for convergence of wired and low-cost wireless technologies in the access network, providing end users with connection flexibility and mobility, is being explored by investigating the interoperability of the multi-PON architecture [2] with WiMAX. This could offer network resilience in case of fibre failure to individual ONUs through the use of overlapping WiMAX cells while allow for efficient dynamic resource allocation to base-station-ONUs of a TDM-PON to provide additional WiMAX channels by means of a centralised signal processing approach in the OLT.

\section{CONCLUSIONS}

The access network architecture presented in this paper utilises the coarse channels of an AWG in the OLT and reflective ONUs to demonstrate dynamic TDM and WDM-PONs, through a single OLT with coarse-fine grooming features. The use of a single-AWG, single-TL OLT to address multiple reflective ONUs of a WDMPON have demonstrated error-free routing of 16, $0.4 \mathrm{~nm}$-spaced wavelengths over a single, 7 nm-wide Gaussian passband window of the AWG in the presence PDW shifting. To manage packet transmission, several DBA protocols have been proposed to dynamically and efficiently arrange the bandwidth among ONUs. Depending on the physical-layer architecture, the innovative DMB protocol has successfully been modified to develop the ADMB and MDMB protocols for TDM and WDM-PONs respectively to efficiently improve the performance of channel throughput and packet delay. Recent developments have concentrated on $100 \mathrm{~km}$-reach networks to achieve comparable network performance in terms of channel utilisation rate, packet delay, and packet loss rate to standard access PONs at a superior $400 \%$ wider network coverage by means of the application of the TSD protocol. Notable initiatives have also been carried out to investigate the application of multiple wavelength operation over standard splitter-based GPONs by extending the dynamic bandwidth algorithms to include an additional dimension, that of wavelength and the integration of WiMAX to terminate wireless users to basestation-ONUs with the intention of providing flexibility in resource allocation among end users.

\section{REFERENCES}

[1] P.-F. Fournier, "From FTTH pilot to pre-rollout in France," presented at CAI Cheuvreux, France, 2007.

[2] Y. Shachaf, C.-H. Chang, P. Kourtessis, J. M. Senior, "Multi-PON access network using a coarse AWG for smooth migration from TDM to WDM PON," OSA Optics Express, vol. 15, pp. 7840-7844, 2007.

[3] C.-H. Chang, P. Kourtessis, and J. M. Senior, "GPON service level agreement based dynamic bandwidth assignment protocol," Journal of Electronics Letters, vol. 42, pp. 1173-1174, 2006.

[4] J. Jiang, C. L. Callender, C. Blanchetière, J. P. Noad, S. Chen, J. Ballato, and J. Dennis W. Smith, "Arrayed Waveguide Gratings Based on Perfluorocyclobutane Polymers for CWDM Applications," IEEE Photonics Technology Letters, vol. 18, pp. 370-372, 2006.

[5] Y. Shachaf, P. Kourtessis, and J. M. Senior, "An interoperable access network based on CWDM-routed PONs," presented at 33rd European Conference and Exhibition on Optical Communication (ECOC), Berlin, Germany, 2007.

[6] C.-H. Chang, P. Kourtessis, and J. M. Senior, "Dynamic Bandwidth assignment for Multi-service access in GPON," presented at 12th European Conference on Networks and Optical Communications (NOC), Stockholm, Sweden, 2007.

[7] G. Kramer, B. Mukherjee, and G. Pesavento, "IPACT a dynamic protocol for an Ethernet PON (EPON)," IEEE Communications Magazine, vol. 40, pp. 74-80, 2002.

[8] R. P. Davey and D. B. Payne, "The future of fibre access systems?" BT Technology Journal, vol. 20, pp. 104 -114, 2002.

[9] C. H. Chang, N. M. Alvarez, P. Kourtessis, and J. M. Senior, "Dynamic Bandwidth assignment for Multi-service access in long-reach GPON," presented at 33rd European Conference and Exhibition on Optical Communication (ECOC), Berlin, Germany, 2007. 